\title{
Study on Regional Economic Growth Led Industrial Development in South Xinjiang
}

\author{
Mei Li ${ }^{1, a}$, Xiaoling Zhu ${ }^{2, b}$ \\ ${ }^{12}$ College of Economics and Management, Tarim University, Alar, XinJiang, 843300 \\ ${ }^{a}$ email, ${ }^{b}$ email
}

Keywords: South Xinjiang; Regional Economy; Urbanization; Industrialization

\begin{abstract}
From the current situation of economic development in the southern border region, due to be affected by geography, climate, history and many other factors, per capita economic level of the region has been in southern China's per capita below the level, and per capita levels in North of there is also a large gap. In order to explore a breakthrough in economic development in southern regions, the author of this article on the impact of the industrial structure of the economy has been explored. The thesis is divided into four parts, the first part of the preface to the study and research methods are described in the second part analyzes the industrial development of three southern states, the third part of the use of gray correlation model of industrial and economic power were analyzed, the fourth model was constructed to predict the development of the industrial economy, and the development of prediction were summarized; fifth segment on the development of industry in the southern border region a number of recommendations, the sixth part of the paper are summarized. With a view to improve references and suggestions for the future development of the southern and better development of the southern industrial economy. Therefore, to discuss constraints Three South urbanization development, and propose appropriate countermeasures for speeding up the development process of urbanization in the region, to ensure the stability and development of border areas are of great practical significance.
\end{abstract}

\section{Introduction}

Introduction: This paper Nanjiang three states, namely Hotan, Kashi Prefecture and the Kirgiz Autonomous Prefecture (hereinafter referred to as g state) as the research object, the main source of data "Xinjiang Statistical Yearbook," the gray related degree method the industrial economy dynamic analysis and evaluation; and build predictive models to forecast and analyze the development of Xinjiang

\section{Industrial Development Situation of the Three States of the Southern Xinjiang}

Since the implementation of the western development strategy, industrial economy Xinjiang region has been greatly improved, but the overall growth rate as compared to other provinces in terms of relatively slow, there is a certain gap. At the same time, because of the South Xinjiang geography, history, natural and social aspects, the level of industrial and economic development Three South overall is still relatively low, compared with the average for the whole of Xinjiang there is a big gap.

We can see that three southern states the proportion of three industries between 2004 and 2011 are relatively stable, relatively small changes in the primary industry, secondary industry are on the rise, the tertiary industry declined. Proportion of primary industry Three South has always accounted for more than $40 \%$, which the whole of Xinjiang and $10 \%$ of industrial production is still mainly in the first industry-based than the first, the three states, three states - because industry underdevelopment and the slow development of the tertiary industry and so increased the dependence of agricultural development.

At present, compared with the northern region, the northern has entered the early industrialization of development, while the southern border is still in the pre-industrial stage of development. In fact, the level of industrialization and urbanization development level has great 
relevance, because improving industrial development level can be a good drive to improve the level of urbanization development, but three southern states to industrialization has been a very backward, causing the level of urbanization development in the southern border region can not be increased. According to statistics, by the end of 2011, the three southern states of the Kirgiz Autonomous Prefecture of urbanization rate was $58.25 \%$, the urbanization rate was $32.9 \%$ in Hotan, Kashgar urbanization rate was $35.4 \%$, and Xinjiang $43.5 \%$ urbanization rate of development there is a big gap.

In this paper, the use of generalized gray incidence model to analyze economic growth, since the model can be intuitive judgments of its close ties based on the geometric sequence acquaintance of the curve, so that can be a big curve in case we can determine the degree of acquaintance association a great degree, otherwise small. Generalized Grey Relational which were gray absolute, relative and overall connection degree.

Sequence from each sequence, etc., can be calculated directly as the starting point of the collar. Initialization formula can be expressed as:

$$
\mathrm{Xi0}=[\mathrm{Xi}(1)-\mathrm{Xi}(1), \mathrm{Xi}(2)-\mathrm{Xi}(1), \ldots, \mathrm{Xi}(\mathrm{T})-\mathrm{Xi}(1)](\mathrm{i}=0,1,2,3)
$$

Where 0 represents the reference sequence area GDP; $1,2,3$ represents the added value of three industries; $\mathrm{T}=12, \mathrm{i}=0,1,2,3$ seek $|\mathrm{Si}|, \quad|\mathrm{S} 0|$ and $\mid \mathrm{Si}-\mathrm{S} 0$. $\mid$,then

$$
\begin{gathered}
\mid \text { Si }|=| \sum_{t=2}^{T-1} x_{i}^{0}(t)+\frac{1}{2} x_{i}^{0}(T)|\quad| \text { So }|=| \sum_{t=2}^{T-1} x_{0}^{0}(t)+\frac{1}{2} x_{0}^{0}(T) \mid \\
\mid \text { Si }- \text { SO }|=| \sum_{t=2}^{T-1}\left[x_{i}^{0}(t)-x_{0}^{0}(t)\right]+\frac{1}{2}\left[x_{i}^{0}(T)-x_{0}^{0}(T)\right] \mid
\end{gathered}
$$

Obtained Gray Correlation $\varepsilon 0 \mathrm{i}(\mathrm{i}=0,1,2,3)=\frac{1+\left|s_{0}\right|+\left|s_{i}\right|}{1+\left|s_{0}\right|+\left|s_{i}\right|+\left|s_{i}-s_{0}\right|}$

The respective number of initialization parameters in accordance with the control column meaning initialization equation can be expressed as:

$$
\begin{gathered}
\left.X_{i}{ }^{\prime}=\left(\frac{X_{i}(1)}{x_{i}(1)}\right), \frac{X_{i}(2)}{x_{i}(1)} \ldots \frac{X_{i}(T)}{x_{i}(1)}\right)(i=0,1,2,3) \\
\left|S_{i}{ }^{\prime}\right|=\mid \sum_{t=2}^{T-1}\left[x_{i}^{0{ }^{\prime}}(t)-\frac{1}{2} x_{i}^{0 \prime}(T)|\quad| S_{0}^{\prime}|=| \sum_{t=2}^{T-1}\left[x_{0}^{0 \prime}(t)-\frac{1}{2} x_{0}^{0 \prime}(T) \mid\right.\right. \\
\left|S_{i}^{\prime}{ }^{\prime} S_{0}{ }^{\prime}\right|=\left|\sum_{t=2}^{T-1}\left[x_{i}^{0 \prime}(t)-x_{0}^{0 \prime}(t)\right]-\frac{1}{2}\left[x_{i}^{0 \prime}(\mathrm{T})+x_{0}^{0 \prime}(\mathrm{T})\right]\right|
\end{gathered}
$$

Obtained Gray Correlation $\gamma 0 \mathrm{i}(\mathrm{i}=0,1,2,3)=\frac{1+\left|s_{0}{ }^{\prime}\right|+\left|s_{i}{ }^{\prime}\right|}{1+\left|s_{0}{ }^{\prime}\right|+\left|s_{i}{ }^{\prime}\right|+\left|s_{i}{ }^{\prime}-s_{0}{ }^{\prime}\right|}$

Finally Grey Synthetic Degree of Grey overall connection degree. $\rho 0 \mathrm{i}=\theta \varepsilon 0 \mathrm{i}+(1-\theta) \gamma 0 \mathrm{i},(=$ $0,1,2,3$, under normal circumstances, $\theta=1 / 2$, Here, $\theta$ takes $1 / 2$.

According to our Xinjiang Statistical Yearbook data, we calculated the years 2000 to 2001 Three South Xinjiang and three industrial added value of the three factors associated with the table of GDP (Table 1 and Table 2)

Table 1 Gray Correlation three southern states of the three industries

\begin{tabular}{cccccccccc}
\hline Area & \multicolumn{2}{c}{ Kashi } & \multicolumn{4}{c}{ Wada } \\
\hline & 1 & 2 & 3 & 1 & 2 & 3 & 1 & 2 & 3 \\
absolute & 0.677 & 0.61 & 0.713 & 0.643 & 0.594 & 0.764 & 0.581 & 0.607 & 0.812 \\
Renative & 0.831 & 0.844 & 0.865 & 0.769 & 0.867 & 0.8 & 0.704 & 0.907 & 0.837 \\
Total & 0.755 & 0.727 & 0.789 & 0.705 & 0.732 & 0.781 & 0.643 & 0.758 & 0.825 \\
\hline
\end{tabular}


Table 2 Grey Correlation Three South Xinjiang and comprehensive three industries

\begin{tabular}{ccccccc}
\hline Area & \multicolumn{2}{c}{ Xinjiang } & \multicolumn{5}{c}{ 3 Area } \\
\hline & 1 & 2 & 3 & 1 & 2 & 3 \\
absolute & 0.583 & 0.753 & 0.657 & 0.662 & 0.606 & 0.732 \\
Renative & 0.895 & 0.897 & 0.925 & 0.81 & 0.852 & 0.846 \\
Total & 0.739 & 0.824 & 0.796 & 0.735 & 0.729 & 0.788 \\
\hline
\end{tabular}

Correlation Table 1 three southern states of the three industries

Kashgar Hotan region Kezhou

A secondary industry and tertiary industries industry industry a secondary industry and tertiary industries industry a secondary industry and tertiary industries

Absolutely 0.6770 .7130 .6430 .610 .5940 .7640 .5810 .6070 .812

0.8310 .8440 .8650 .7690 .867 Relative 0.80 .7040 .9070 .837

Comprehensive 0.7550 .7270 .7890 .7050 .7320 .7810 .6430 .7580 .825

Grey Correlation Table 2 Three South Xinjiang and comprehensive three industries

Areas of three states

Industry Category primary industry secondary industry and tertiary industries industry a secondary industry and tertiary industries

0.5830 .7530 .6570 .6620 .606 absolutely 0.732

Relative 0.8950 .8970 .9250 .810 .8520 .846

Composite Index 0.7390 .8240 .7960 .7350 .7290 .788

From Figure 2 we can see that the second industry association of Xinjiang GDP and is the largest, followed by the tertiary industry, and finally the primary sector. This makes it possible that the industrial structure of Xinjiang industry as the leading force in the second degree of relevance of GDP the second largest industry in Xinjiang, Xinjiang's economic growth stimulating effect is greatest. The associate degree from the total value of the tertiary industry and production in Figure III Three South is the largest, followed by the primary industry, secondary industry finally. Thus, you can learn three southern states mainly in the tertiary industry as the leading, Xinjiang and the development of secondary industry average in terms of relatively backward, pulling effect on the economy is not obvious.

\section{Three South Xinjiang Industrial Economic Development Forecasting Model}

In order to do well on the industry forecast, the author constructed the gray dynamic forecasting model, mainly based on GM $(1,1)$ model of gray system theory is built. Because the prediction model consists of a single variable first-order differential equations, when will this model fitting and prediction for a dominant factor when complex systems, can be a good development trend and predict the variation of the dominant factors. Therefore, in the industrial and economic development of the southern states of the three judges, the author also uses gray system GM $(1,1)$ forecasting model to predict the dynamic.

First, we need to build predictive models of before, we need to do to verify the model quasi smoothness and quasi exponentially:

(1) quasi-smooth test, the original series $\mathrm{Y}(0)(\mathrm{k})$ accumulating derived a summing operator $\mathrm{Y}(1)$ $(\mathrm{k})$, to test the original series $\mathrm{Y}(0)$ of the equation is expressed as: $\rho(\mathrm{k})=\mathrm{Y}(0)(\mathrm{k}) / \mathrm{Y}(1)(\mathrm{k}-1)$, for $\rho(\mathrm{k})$ values are calculated, if $\mathrm{k}>3, \rho(\mathrm{k})<0.5$, then the quasi-smooth conditions are met, and vice versa not eligible.

(2) quasi-exponential test, the formula of specific tests is expressed as: $\sigma(1)(\mathrm{k})=\mathrm{Y}(1)(\mathrm{k}) / \mathrm{Y}(1)$ $(\mathrm{k}-1)$, for $\sigma(1)(\mathrm{k})$ values are calculated, if $\mathrm{k}>3, \sigma(1)(\mathrm{k}) \in[1,1.5], \delta<0.5$, in line with the quasi-exponential, not vice versa accord.

Then, when two or more test steps are passed, we also need to examine the accuracy of the model, and the applicable conditions of the model study, if the model can be used as short-term or long-term forecast, the model can be used prediction: 
(1) test the model's accuracy. Residual test method can use the method of model accuracy tests, generally speaking, the relative valuation model residual error is less than $5 \%$, when different issues have different considerations $10 \%$, given the ecological indicators when the value of a large gap, so the average is less than $10 \%$ relative error of the model selected in this residual valuation, the detection accuracy of the model in line.

(2) model applicable conditions investigated. If the development of the model coefficient is less than 0.3, then the model as a long term forecasting model; if the development of the model coefficients greater than 0.3 less than or equal to 0.5 , the change only as a short-term forecasting model based on the judgment, but as a long-term forecast We need to be careful; if the development of the model coefficients greater than 0.5 less than or equal to 0.8 , then the short-term forecast also need to be very cautious.

By "Xinjiang Statistical Yearbook" data, construct and gray association model and gray prediction model for the relationship between the three industrial structure and the GDP of Xinjiang and three southern states to analyze and predict, we summed up the following two conclusions:

(1) the overall industrial and economic development Three South is still relatively backward, still agriculture-led economy. Three southern states with 30 percent of Xinjiang's population and 28\% of the land area of Xinjiang to create only 9 percent of gross economic output of Xinjiang. Self-development capacity is weak, low level of economic development in Xinjiang and the average there is a big gap, plus modernization, industrialization and urbanization are relatively slow development of agriculture, further hampered economic growth in southern Xinjiang.

(2) the end of the second industry in Southern stimulating effect on economic growth, industrial development has lagged behind the primary industry, the tertiary industry also contributed to economic growth is greater than the secondary industry and the tertiary industry accounted relatively high write. Found mainly in southern commerce and other traditional services and public services as the main source of economic development, but also the need to strengthen the development of the secondary industry, the development of modern agriculture also needs to accelerate.

\section{Three South Xinjiang Future Industrial Development Countermeasures and Suggestions}

In order to better promote the increased role of the secondary industry in the economy, the southern border region should increase efforts to develop industry and accelerate the development of the southern border region into the middle period of industrialization. Therefore, we can use the resources of fruit and cotton in southern Xinjiang region has the resources, efforts to develop fruit-based processing industry and the textile industry, the use of bounteous land area, the introduction of foreign enterprises to develop production and assembly processing industries.

In order to better promote the modernization of agriculture and animal husbandry development, and innovation have the characteristics of southern agriculture, therefore, should be introduced in Southern agricultural modernization technologies, to promote the development of agricultural industrialization, the southern border region to improve water conservancy, roads and other facilities infrastructure, focusing on the development of agriculture, farming and horticulture and the like.

Southern Xinjiang region accounts for about 30\% of the population, but the majority of the rural population, the poor are concentrated. Thus, the southern border region should speed up the migration of the rural population, promoting the construction of new urbanization. For example, the transformation of old housing, construction of affordable housing, accelerate the construction of housing for low-income people to protect, improve the urban infrastructure and public service facilities construction, the guide of the rural population to migrate to small towns, so that workforce is concentrated in the cities and towns, promote secondary industry development, construction have special southern small towns like.

In order to better develop the southern border region of the tertiary industry, the southern border region should be to improve the quality of the tertiary industry based, accelerate the development of modern service industry. For example, the southern border region can rely on the southern border of 
the unique natural scenery, ethnic customs, historical and cultural landscape of jade and other resources, the development of the tourism sector in Southern culture, improve community health, culture and entertainment, domestic cleaning, childcare and pension quality of food distribution and other services.

\section{Conclusion}

An Empirical Analysis by Southern Xinjiang Industrial Structure made to the development of industry and the industrial structure and the current situation around the state Nanjiang an in-depth understanding, we can see that the evolution of the industrial structure has a great contribution to the economic growth of the association, because bit better so that the coordinated development of the regional industry, a variety of industries and jointly promote the economic development of southern Xinjiang, the southern border region should vigorously develop modern agriculture and animal husbandry industry, the development of distinctive, promote the coordinated development of industrialization and urbanization, improve the modern quality of service.

\section{Acknowledgements}

Fund Project: National Social Science Fund Project "transformation of economic development under the background of the coordinated development of southern Xinjiang Urbanization and Industrial Research (item number: 12XJY004)

\section{References}

[1] Lu Xincong. the status quo of urbanization Zhu Xiaoling three southern states and hinder factors [J] Rural Economy and Science, 2013,04: 98-99 + 97.

[2] Bai Xuemei. regional economic development in China comparative study [M] Beijing: China Financial and Economic Publishing House, 1998.

[3] Qin Chunyan. Three South Xinjiang Economic Development Status and Trends [J]. Finance \& Economics, 2013 (05).

[4] Yuan Xiaojun, Sun Jinshan. empirical analysis of industrial structure and economic growth in Xinjiang [J]. Commercial Times, 2013 (11).

[5] Xia Hongbao. Road urbanization industrialization drive innovation and development [J]. Theory of Urban Construction, 2013 (34).

[6] Wang Chenjun, Zhang Tian. Prediction based real estate development value GM $(1,1)$ model [J]. Commercial Times, 2010 (05). 